\title{
Variability and inter relationship studies in tomato (Solanum lycopersicum L.)
}

\author{
M. Z. Ullah*, L. Hassan, Sonia Binte Shahid ${ }^{1}$ and A. K. Patwary \\ Department of Genetics and Plant Breeding, Bangladesh Agricultural University, Mymensingh-2202 and \\ ${ }^{1}$ Department of Plant and Environmental Biotechnology, Sylhet Agricultural University, Sylhet, \\ Bangladesh, *E-Mail: zahirsau@gmail.com
}

\begin{abstract}
Twenty parental genotypes of tomato were planted and observed of yield and yield attributing traits to measure genetic variability, character association and path coefficient analysis. Parent TM 371 ranked first with respect to yield (4.73 kg fruit yield per plant) followed by TM 390 . In terms of average fruit weight TM 390 also was better performer. Analysis of variance for each trait showed significant differences among the genotypes. High genotypic and phenotypic coefficients of variation were recorded for fruits per plant, locule number per fruit and fruit yield per plant. Heritability was observed high for flowers per cluster, fruits per plant, fruit weight and fruit length. fruit per plant (52.30), followed by fruit weight (46.32). High heritability associated with high genetic advance was observed for fruits per plant (52.30) and fruit weight (46.32) and flower per cluster (33.50). Selection for such traits might be effective for the fruit yield improvement of tomato. Significant positive genotypic and phenotypic correlation was observed of fruits per plant, fruit weight, fruit diameter and locule number per fruit with fruit yield per plant indicated that selection strategies must be focus on these traits. Fruit diameter showed the highest positive direct effect (3.25) on fruit yield per plant followed by fruits per plant (1.54). Direct selection may be executed considering these traits as the main selection criteria to reduce indirect effect of the other characters during the development of high yielding tomato variety.
\end{abstract}

Keywords: Variability, Correlation, Genetic advance, Heritability

\section{Introduction}

Tomato is one of the most important and popular winter vegetable in Bangladesh. Tomato is an introduced crop in Bangladesh and provides less genetic variability. It is estimated that the genomes of tomato cultivars contain $<5 \%$ of the genetic variation of their wild relatives. Since the 20 th century, human beings have created a huge array of morphologically different cultivars and forms from the single species S. Iycopersicum via plant breeding. Through domestication, research and breeding activities that were implemented by scientists and breeders worldwide, modern tomato varieties (mostly hybrids) have been developed with all shapes, colors and sizes.

(Bai and Lindhot, 2007). In Bangladesh most of the tomato varieties are of inbred type, those are low yielder. Average yield of tomato is very low (7.51 t/ha) in Bangladesh compared to other tropical countries (15.1 t/ha in India) in the world (Anon, 2004).

Variability in tomato is expected to be immense as the fruits vary greatly in shape and size (Dixit and Dubey, 1985; Bhardwaj and Sharma, 2005). Studies on genetic parameters and character associations provide to select and help to develop optimum breeding procedure. Many researchers (Kamruzzahan et al., 2000) have reported different genetic parameters in tomato based on few traits. As yield is the main object of a breeder, it is important to know the relationship between various characters that have direct and indirect effect on yield. The degree of relationship or association of these characters with yield can be ascertained by correlation studies. This would aid in formulating an efficient breeding program for improving the yield potential via its components (Frageria and Kokli, 1997). Considering all the facts described above the present investigation was undertaken with the following objectives: (1) To estimate genetic variability of inbreed parental lines(2) To study the character association and (3) To study path coefficient analysis showing direct and indirect effects 


\section{Materials and Methods}

Twenty genotypes of tomato comprising fifteen female and five male parents were evaluated in RCBD in three replications during Rabi season 2012at Research \& Development Farm of Energypac Agro Ltd., Gazipur. The observations were taken on ten competitive plants from each replicationfor days to $50 \%$ flowering, flowers per cluster, fruits per cluster, fruits per plant, plant height, fruit weight, fruit length, fruit diameter, pericarp thickness, locule number and fruit yield per plant. The collected pool data were subjected for statistical analysis as per the methodof Panse and Sukhatme (1978). Genotypic and phenotypic coefficient of variances (GCV \&PCV) was calculated according to Burton and De Vane (1953). Heritability and genetic advance were calculated according to Hanson et al. (1956) and Johnson et al. (1955), respectively. The correlation coefficient was estimated according to formulae of Al-Jibouri et al. (1958).

\section{Results and Discussion}

Twenty genotypes of tomato were evaluated for eleven yield and yield contributing characters to work out the extent of variability, correlation, heritability and genetic gain (Table 1). Parent TM 371 ranked $1^{\text {st }}$ with $4.73 \mathrm{~kg}$ fruit yield per plant followed byTM 390. In terms of fruit weight, parents TM 390 was better having average fruit weight of $121.63 \mathrm{~g}$ TM 392, TM 422, TM 409 and TM 419 were other varieties having fruit weight in the desirable range.

Table 1. Phenotypic attributes of tomato varieties

\begin{tabular}{|c|c|c|c|c|c|c|c|c|c|c|c|c|}
\hline Genotypes & $\mathrm{D} 50 \% \mathrm{~F}$ & FPC & FRPC & FPP & $\mathrm{PH}(\mathrm{cm})$ & $F W(g)$ & $\mathrm{FL}(\mathrm{cm})$ & $\mathrm{FD}(\mathrm{cm})$ & $\mathrm{PT}(\mathrm{mm})$ & $\mathrm{LN}$ & FYP (Kg) & Rank \\
\hline \multicolumn{13}{|l|}{ Female } \\
\hline \begin{tabular}{|l|} 
TM 356 \\
\end{tabular} & 54.33 & 6.72 & 4.73 & 29.66 & 114.33 & 63.19 & 4.56 & 5.47 & 6.83 & 2.67 & 1.84 & $\mathrm{XIX}$ \\
\hline TM 361 & 61.66 & 6.82 & 5.08 & 58.66 & 118.33 & 53.89 & 4.33 & 4.57 & 6.83 & 2.00 & 3.62 & IV \\
\hline TM 368 & 54.33 & 6.02 & 4.62 & 43.66 & 78.00 & 83.80 & 5.43 & 6.63 & 8.00 & 3.67 & 4.25 & III \\
\hline TM 371 & 57.66 & 5.19 & 4.16 & 59.66 & 81.33 & 89.91 & 5.43 & 6.00 & 6.97 & 4.33 & 4.73 & I \\
\hline TM 377 & 62.33 & 5.10 & 4.55 & 32.33 & 67.67 & 65.96 & 5.23 & 5.40 & 7.00 & 2.33 & 2.12 & XVIII \\
\hline TM 382 & 57.00 & 5.29 & 4.01 & 36.67 & 97.00 & 89.53 & 5.06 & 6.27 & 7.00 & 4.33 & 3.25 & $\mathrm{XI}$ \\
\hline TM 384 & 56.33 & 7.73 & 6.10 & 36.67 & 78.33 & 73.04 & 5.66 & 5.33 & 7.00 & 2.67 & 2.67 & $\mathrm{XV}$ \\
\hline TM 388 & 62.00 & 5.43 & 4.05 & 39.67 & 88.67 & 85.25 & 5.40 & 5.70 & 7.33 & 3.00 & 3.54 & $\mathrm{Vl}$ \\
\hline TM 390 & 59.66 & 4.66 & 3.83 & 35.33 & 68.33 & 121.63 & 5.23 & 6.40 & 7.13 & 4.33 & 4.44 & II \\
\hline TM 392 & 56.33 & 4.64 & 4.32 & 28.67 & 71.67 & 115.72 & 5.63 & 6.27 & 6.33 & 3.67 & 3.50 & VIII \\
\hline TM 410 & 68.33 & 5.79 & 4.90 & 41.33 & 97.67 & 83.16 & 5.40 & 5.77 & 6.67 & 3.00 & 3.40 & $X$ \\
\hline TM 419 & 56.00 & 6.41 & 5.08 & 23.33 & 76.67 & 95.33 & 5.63 & 5.77 & 7.33 & 4.00 & 2.21 & $\overline{X V I}$ \\
\hline TM 422 & 63.33 & 4.04 & 2.54 & 33.33 & 125.33 & 102.24 & 5.46 & 5.93 & 7.83 & 3.33 & 3.44 & IX \\
\hline TM 423 & 60.66 & 5.76 & 3.59 & 41.00 & 102.33 & 71.99 & 5.06 & 6.20 & 6.50 & 5.33 & 3.23 & XII \\
\hline TM 360 & 64.66 & 6.50 & 4.60 & 41.33 & 88.67 & 76.76 & 4.66 & 5.10 & 7.67 & 3.67 & 3.21 & XIII \\
\hline \multicolumn{13}{|l|}{ Male } \\
\hline TM 349 & 60.00 & 6.36 & 3.93 & 40.33 & 61.33 & 77.26 & 5.80 & 4.93 & 9.90 & 2.00 & 3.13 & XIV \\
\hline TM 528 & 62.00 & 8.21 & 6.44 & 29.67 & 85.67 & 75.00 & 5.93 & 5.33 & 8.67 & 2.00 & 2.17 & XVII \\
\hline TM 403 & 53.00 & 4.41 & 3.86 & 75.33 & 88.33 & 49.00 & 5.23 & 4.40 & 6.33 & 2.67 & 3.57 & $\mathrm{~V}$ \\
\hline TM 386 & 70.00 & 7.13 & 4.28 & 33.00 & 91.00 & 39.36 & 6.26 & 4.03 & 6.77 & 2.00 & 1.29 & $X X$ \\
\hline TM 409 & 59.66 & 6.54 & 5.01 & 37.00 & 75.33 & 100.76 & 7.26 & 5.33 & 7.33 & 2.67 & 3.51 & VII \\
\hline
\end{tabular}

Analysis of variance indicated that highly significant variation was observed among the studied parental lines(Table 2). Range indicating high variability for fruits per plant, plant height, fruit weight and fruit yield per plant. High genotypic and phenotypic coefficients of variation were recorded for fruits per plant, locule number per fruit and fruit yield per plant. Heritability was observed high for flowers per cluster, fruits per plant, fruit weight and fruit length. High heritability associated with high genetic advance was observed for fruits per plant (52.30) and fruit weight (46.32) and flower per cluster (33.50). Mohanty (2003)reported high genotypic coefficient of variation and heritability in 18 tomato varieties. Vikram and Kohli (1998) in their study on 25 tomato genotypes recorded high heritability and genetic advance for mean fruit weight and suggested that improvement for fruit weight is possible by simple selection method. Similar results were also reported by Singh and Narayan (2004) in an investigation conducted on 10 tomato varieties. 
Table 2. Estimation of genetic parameters in eleven characters of 20 genotypes in tomato

\begin{tabular}{|l|c|c|c|c|c|c|c|c|c|c|}
\hline Parameters & Range & Mean & MS & CV (\%) & PCV & GCV & ECV & Heritability & $\begin{array}{c}\text { Genetic } \\
\text { advance } \\
\text { (5\%) }\end{array}$ & $\begin{array}{c}\text { Genetic } \\
\text { advance } \\
\text { (\% mean) }\end{array}$ \\
\hline D50\%F & $50.00-72.00$ & 59.96 & $61.54^{\star *}$ & 5.67 & 8.86 & 6.81 & 5.67 & 59.03 & 6.46 & 10.77 \\
\hline FPC & $3.67-9.13$ & 5.94 & $3.71^{\star *}$ & 8.71 & 20.04 & 18.04 & 8.72 & 81.08 & 1.99 & 33.50 \\
\hline FRPC & $2.33-6.67$ & 4.48 & $2.20^{\star *}$ & 12.45 & 21.65 & 17.71 & 12.45 & 66.92 & 1.34 & 29.91 \\
\hline FPP & $18.00-84.00$ & 39.83 & $446.26^{\star *}$ & 16.38 & 33.41 & 29.12 & 16.38 & 75.97 & 20.83 & 52.30 \\
\hline PH & $59.00-159.00$ & 87.80 & $901.06^{\star *}$ & 13.37 & 22.56 & 18.17 & 13.37 & 64.86 & 26.46 & 30.14 \\
\hline FW & $37.10-131.30$ & 80.64 & $1315.58^{\star *}$ & 12.16 & 27.80 & 25.00 & 12.16 & 80.86 & 37.35 & 46.32 \\
\hline FL & $4.20-7.60$ & 5.43 & $1.18^{\star *}$ & 4.11 & 12.05 & 11.32 & 4.11 & 88.35 & 1.19 & 21.92 \\
\hline FD & $3.80-7.80$ & 5.54 & $1.44^{\star *}$ & 9.17 & 14.59 & 11.35 & 9.17 & 60.48 & 1.01 & 18.23 \\
\hline PT & $5.50-10.00$ & 7.27 & $2.14^{\star *}$ & 9.92 & 14.16 & 10.11 & 9.92 & 50.96 & 1.08 & 14.86 \\
\hline LN & $2.00-6.00$ & 3.18 & $2.75^{\star *}$ & 24.14 & 35.97 & 26.67 & 24.14 & 54.98 & 1.30 & 40.88 \\
\hline FYP & $1.00-5.80$ & 3.16 & $2.31^{* *}$ & 19.47 & 32.03 & 25.42 & 19.48 & 63.00 & 1.31 & 41.46 \\
\hline
\end{tabular}

**, * Correlation is significant at the 0.01 and 0.05 level, respectively.

MS = mean sum of square, $\mathrm{CV}(\%)=$ Coefficient of variation, PCV = Phenotypic Coefficient of Variation, GCV $=$ Genotypic Coefficient of Variation and ECV = Environmental Coefficient of Variation, D50\%F= Days to 50\% flowering, FPC $=\mathrm{Flower}$ per luster, $\mathrm{FRPC}=$ Fruit per cluster, $\mathrm{FPP}=$ Fruit per plant, $\mathrm{PH}=$ Plant height $(\mathrm{cm}), \mathrm{FW}=$ Fruit weight $(\mathrm{g}), \mathrm{FL}=\mathrm{Fruit}$ length $(\mathrm{cm}), \mathrm{FD}=\mathrm{Fruit}$ diameter $(\mathrm{cm}), \mathrm{PT}=$ Pericarp thickness $(\mathrm{mm}), \mathrm{LN}=$ Locule number per fruit and FYP= Fruit yield per plant $(\mathrm{Kg})$.

Character association: Association analysis for five yield-contributing attributes revealed that genotypic correlations were higher than phenotypic correlations in general (Table 3). Similar findings were reported by Yadav and Singh (1998) in a study on 28 tomato varieties stated that genotypic correlations were higher than phenotypic correlations. Days to $50 \%$ flowering showed negative relationship with fruit diameter at both genotypic and phenotypic level and significantly negative phenotypic correlation with locule number. Flower per cluster was significantly and positively associated with fruits per cluster but negative significantly associated with fruit weight, fruit diameter and locule number per fruit. Highly significant negative correlation was observed of fruits per plant with fruit weight at both levels. Plant height had significantly negative correlation with fruit length at both level and significant genotypic correlation with fruit weight and pericarp thickness but non-significantly positive correlation with fruits per plant. Significant positive relationship was observed fruit weight with fruit diameter and locule number per fruit. Fruit diameter has positive significant association with locule number per fruit. Significant negative relationship of pericarp thickness with locule number per fruit. Finally fruit yield was positively significant associated with fruits per plant, fruit weight, fruit diameter and locule number per fruit. Similar result shave also been reported by Agong et al. (2008), Haydar et al. (2007), Mohanty (2003), Harer et al.(2003), Mohanty (2002a), Mohanty (2002b) intomato.

Path Coefficient Analysis: Fruit diameter showed the highest positive direct effect (3.25) on fruit yield per plant followed by fruits per plant (1.54) (Table 4). Days to $50 \%$ flowering also showed positive direct effect on fruit yield per plant. On the other hand, negative direct effect on yield per plant showed by fruit weight, locule number per fruit and flower per cluster. Fruits per plant, fruit weight, fruit diameter and locule number per fruit showed significant positive genotypic correlation with fruit yield per plant. The highest indirect effect of fruit weight was observed with fruit diameter. The characters showing high direct effect on yield per plant indicated that direct selection for these traits might be effective and there is a possibility of improving yield per plant through selection based on these characters. Similar results have also been reported by Dhankar et al. (2001), Verma and Sarnaik (2000), Mageswari et al. (1999), Prasad and Rai (1999), Yadav and Singh (1998), Singh et al. (1997). 
Table 3. Genotypic (G)and phenotypic (P) correlation coefficients among different pairs of yield and yield contributing characters for different genotype of mustard

\begin{tabular}{|c|c|c|c|c|c|c|c|c|c|c|c|}
\hline Characters & & FPC & FRPC & FPP & $\mathrm{PH}$ & FW & $\mathrm{FL}$ & FD & PT & LN & FYP \\
\hline \multirow{2}{*}{$\mathrm{D} 50 \% \mathrm{~F}$} & G & 0.226 & -0.078 & -0.213 & 0.247 & -0.216 & 0.147 & $-0.364^{\star *}$ & 0.097 & $-0.352^{* *}$ & $-0.313^{*}$ \\
\hline & $P$ & 0.084 & -0.032 & -0.172 & 0.134 & -0.165 & 0.126 & $-0.286^{*}$ & 0.047 & -0.158 & -0.207 \\
\hline \multirow{2}{*}{ FPC } & G & & $0.930^{* *}$ & $-0.270^{*}$ & -0.063 & $-0.480^{* \star}$ & 0.197 & $-0.475^{\star \star}$ & $0.344^{\star \star}$ & $-0.520^{\star \star}$ & $-0.637^{\star *}$ \\
\hline & $P$ & & $0.645^{\star \star}$ & -0.221 & 0.028 & $-0.343^{\star *}$ & 0.184 & $-0.312^{*}$ & 0.251 & $-0.387^{* *}$ & $-0.394^{* *}$ \\
\hline \multirow{2}{*}{ FRPC } & G & & & -0.231 & $-0.260^{*}$ & -0.227 & 0.174 & -0.238 & 0.064 & $-0.503^{\star *}$ & $-0.413^{* *}$ \\
\hline & $P$ & & & -0.115 & -0.198 & -0.173 & 0.168 & -0.171 & 0.106 & $-0.302^{*}$ & $-0.270^{*}$ \\
\hline \multirow{2}{*}{ FPP } & $G$ & & & & 0.156 & $-0.383^{\star *}$ & $-0.292^{*}$ & $-0.363^{\star *}$ & $-0.276^{*}$ & -0.035 & $0.528^{\star *}$ \\
\hline & $P$ & & & & 0.124 & $-0.373^{\star \star}$ & -0.234 & -0.249 & -0.129 & -0.032 & $0.529^{\star *}$ \\
\hline \multirow{2}{*}{$\mathrm{PH}$} & G & & & & & $-0.376^{* *}$ & $-0.533^{* *}$ & -0.161 & $-0.331^{\star *}$ & 0.050 & -0.216 \\
\hline & $\mathrm{P}$ & & & & & -0.179 & $-0.360^{\star *}$ & -0.043 & -0.228 & -0.040 & 0.053 \\
\hline \multirow{2}{*}{ FW } & G & & & & & & 0.245 & $0.861^{* *}$ & 0.165 & $0.614^{* *}$ & $0.551^{* *}$ \\
\hline & $P$ & & & & & & 0.183 & $0.644^{* *}$ & 0.033 & $0.426^{* *}$ & $0.494^{* *}$ \\
\hline \multirow{2}{*}{$\mathrm{FL}$} & G & & & & & & & -0.138 & 0.251 & $-0.258^{\star}$ & -0.134 \\
\hline & $P$ & & & & & & & -0.028 & 0.216 & -0.185 & -0.098 \\
\hline \multirow{2}{*}{ FD } & G & & & & & & & & -0.050 & $0.849 * *$ & $0.583^{\star *}$ \\
\hline & $P$ & & & & & & & & -0.026 & $0.635^{\star *}$ & $0.393^{\star \star}$ \\
\hline \multirow[t]{2}{*}{ PT } & G & & & & & & & & & $-0.314^{*}$ & -0.042 \\
\hline & $P$ & & & & & & & & & $-0.303^{*}$ & -0.005 \\
\hline \multirow[t]{2}{*}{ LN } & G & & & & & & & & & & $0.612^{\star *}$ \\
\hline & $P$ & & & & & & & & & & $0.330^{*}$ \\
\hline
\end{tabular}

Table 3. Path coefficient analysis showing direct and indirect effects of different characters on yield of mustard

\begin{tabular}{|l|c|c|c|c|c|c|c|c|c|c|c|c|}
\hline Characters & $\begin{array}{c}\text { Direct } \\
\text { effect }\end{array}$ & D50\%F & FPC & FRPC & FPP & PH & FW & FL & FD & PT & LN & $\begin{array}{c}\text { Genotypic correlation } \\
\text { with yield }\end{array}$ \\
\hline D50\%F & 0.53 & - & -0.06 & -0.01 & -0.33 & 0.00 & 0.30 & 0.11 & -1.18 & 0.03 & 0.30 & $-0.313^{*}$ \\
\hline FPC & -0.27 & 0.12 & - & 0.10 & -0.41 & 0.00 & 0.66 & 0.15 & -1.55 & 0.12 & 0.44 & $-0.637^{\star *}$ \\
\hline FRPC & 0.11 & -0.04 & -0.25 & - & -0.35 & 0.00 & 0.31 & 0.13 & -0.77 & 0.02 & 0.43 & $-0.413^{\star *}$ \\
\hline FPP & 1.54 & -0.11 & 0.07 & -0.03 & - & 0.00 & 0.53 & -0.22 & -1.18 & -0.10 & 0.03 & $0.528^{\star *}$ \\
\hline PH & -0.01 & 0.13 & 0.02 & -0.03 & 0.24 & - & 0.52 & -0.40 & -0.52 & -0.12 & -0.04 & -0.216 \\
\hline FW & -1.38 & -0.11 & 0.13 & -0.03 & -0.59 & 0.01 & - & 0.18 & 2.80 & 0.06 & -0.52 & $0.551^{\star *}$ \\
\hline FL & 0.74 & 0.08 & -0.05 & 0.02 & -0.45 & 0.01 & -0.34 & - & -0.45 & 0.09 & 0.22 & -0.134 \\
\hline FD & 3.25 & -0.19 & 0.13 & -0.03 & -0.56 & 0.00 & -1.19 & -0.10 & - & -0.02 & -0.72 & $0.583^{\star *}$ \\
\hline PT & 0.35 & 0.05 & -0.09 & 0.01 & -0.42 & 0.00 & -0.23 & 0.19 & -0.16 & - & 0.27 & -0.042 \\
\hline LN & -0.85 & -0.19 & 0.14 & -0.06 & -0.05 & 0.00 & -0.85 & -0.19 & 2.76 & -0.11 & - & $0.612^{\star *}$ \\
\hline
\end{tabular}

Residual effect: 0.423

* = Significant at $5 \%$

$\star \star=$ Significant at $1 \%$. 
Ullah et al.

\section{References}

Agong, S.G., Schittenhelm, S. and Friedt, W. 2008. Genotypic variation of Kenyan tomato (Lycopersicon esculentum L.) germplasm. PGR Newsletter, FAO Biodiversity 123: 61-67.

Al-Jibouri, H.A., Millar, P.A. and Robinson, H.F. 1958. Genotypic and environmental variances and co-variances in an upland cotton cross of inter-specific origin. Agron. J., 50: 633- 636.

Anonymous. 2004. Yearbook of Agricultural Statistics. Bangladesh Bureau of Statistics, Statistics Division, Ministry of Planning, Dhaka, Bangladesh, p 111.

Bai Y., Lindhout, P 2007. Domestication and Breeding of Tomatoes: What Have We Gained and What Can We Gain in the Future. Annals of Botany 100 (5) 1085-1094.

Bhardwaj, N.V., Sharma, M.K. 2005: Genetic parameters and character association in tomato. Bangladesh Journal of Agricultural Research 30 (1) 49-56.

Burton, G.W. and De Vane, E. 1953. Estimating heritability in tall fescue (Festuca arundinacea). Agron. J., 45: 478-481.

Dhankar, S.K., Dhankhar, B.S., Sharma, N.K. 2001. Correlation and path analysis in tomato under normal and high temperature conditions. Haryana Journal of Horticultural Sciences 30 (1-2): 89-92.

Dixit P. and Dubey, D.K. 1985. Heritability and genetic advance in induced mutant in lentil. Ind J Genet 45 (3): 520-524.

Frageria, M.S. and Kokli, U.K. 1997. Correlation studies in tomato. Haryana J Hortic Sci 25: 158- 160.

Hanson, C.H., Robinson, H.F. and Comstock, R.E. 1956. Biometrical studies of yield in segregating population of Korean lespedeza. Agron. J., 47: 268-272.

Harer, P.N., Lad, D.B. and Bhor, T.J. 2003. Correlaton and path analysis studies in tomato. J. Maharashtra Agril. Univ. 27: $302-303$.

Haydar, A., Mandal, M.A., Ahmed, M.B., Hannan, M.M., Karim, R., Razvy, M.A., Roy, U.K. and Salahin, M. 2007. Studies on genetic variability and interrelationship among the different traits in tomato. Middle East Journal of Scientific Research 2 (3-4): 139-142.

Johnson, H.W., Robinson, H.F. and Comstock, R.E. 1955. Estimates of genetic and environmental variability in soyabean. Agron. J., 47: $34-38$

Kamruzzahan, M., Hossain, M., Islam, R. and Alam, M.F. 2000. Variability and correlation studies in tomato (Lycopersicon esculentum Mill.). Bangladesh J Genet Biotech 1 (1): 21-26.

Mageswari, K., Natarajan, S. and Thamburaj, S. 1999. Causal influence of component traits on yield. Maharashtra Agricultural Universities 27 (3): 302-303.

Mohanty, B.K. 2002a. Variability, heritability, correlation and path coefficient studies in tomato. Haryana Journal of Horticultural Sciences 31 (3-4): 230-233.

Mohanty, B.K. 2002b. Studies on variability, heritability, interrelationship and path analysis in tomato. Annals of Agricultural Research 23 (1): 65-69.

Mohanty, B.K. 2003. Genetic variability, correlation and path coefficient studies in tomato. Indian Journal of Agricultural Research 37 (1): 68-71.

Phanse, V.G. and Sukhatme, P.V. 1978. Statistical Methods for Agricultural Workers. ICAR, New Delhi, pp: 68-75.

Prasad, V.S.R.K. and Rai, M. 1999. Genetic variation, component association and direct and indirect selections in some exotic tomato germplasm. Indian Journal of Horticulture 56 (3): 262-266.

Singh, A.K. and Narayan, R. 2004. Variability studies in tomato under Cold Arid Condition of Ladakh. The Hort. J . 17: 67-72.

Singh, D.N., Sahu, A. and Parida, A.K. 1997. Genetic variability and correlation studies in tomato (Lycopersicon esculentum Mill.). Environment and Ecology 15 (1): 117-121.

Verma, S.K. and Sarnaik, D.A. 2000. Path analysis of yield components in tomato (Lycopersicon esculentum Mill). Journal of Applied Biology 10 (2): 136-138.

Vikram, A and Kohli, U.K. 1998. Genetic variability, correlation and path analysis in tomato. J. Hill Res. 11: 107-111.

Yadav, D.S. and Singh, S.P. 1998. Correlation and path analysis in tomato. J. Hill Res. 11: 207-211. 